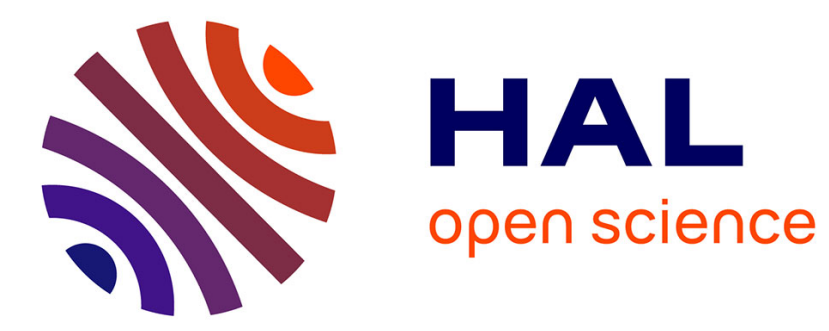

\title{
In-plane propagation of excitonic cavity polaritons
}

\author{
T. Freixanet, B. Sermage, A. Tiberj, R. Planel
}

\section{To cite this version:}

T. Freixanet, B. Sermage, A. Tiberj, R. Planel. In-plane propagation of excitonic cavity polaritons. Physical Review B: Condensed Matter and Materials Physics (1998-2015), 2000, 61 (11), 10.1103/PhysRevB.61.7233 . hal-01213439

\section{HAL Id: hal-01213439 \\ https://hal.science/hal-01213439}

Submitted on 8 Oct 2015

HAL is a multi-disciplinary open access archive for the deposit and dissemination of scientific research documents, whether they are published or not. The documents may come from teaching and research institutions in France or abroad, or from public or private research centers.
L'archive ouverte pluridisciplinaire HAL, est destinée au dépôt et à la diffusion de documents scientifiques de niveau recherche, publiés ou non, émanant des établissements d'enseignement et de recherche français ou étrangers, des laboratoires publics ou privés. 


\title{
In-plane propagation of excitonic cavity polaritons
}

\author{
T. Freixanet \\ Laboratoire de Microstructures et de Microélectronique, Bôite Postale 107, 92225 Bagneux, France \\ B. Sermage and A. Tiberj \\ Centre National d'Etudes des Télécommunications, Boite Postale 107, 92225 Bagneux, France \\ R. Planel \\ Laboratoire de Microstructures et de Microélectronique, Bôite Postale 107, 92225 Bagneux, France
}

(Received 16 November 1999)

\begin{abstract}
By exciting resonantly a microcavity with a pulsed laser beam, we have observed the in-plane propagation of excitonic cavity polaritons over distances much greater than the wavelength. The initial in-plane wave vector is conserved during the propagation, showing that it is a good quantum number for cavity polaritons. The polariton group velocities were measured as a function of both the in-plane wave vector and detuning. Propagation is faster for negative detunings where the polaritons have a light effective mass. The observations are in good agreement with a simple two-oscillator model.
\end{abstract}

Physicists were very enthusiastic when they could reproduce in semiconductor microcavities ${ }^{1}$ the strong coupling regime between light and matter predicted by QED (quantum electrodynamics theory) and usually observed in atomic physics. $^{2}$ One of its most spectacular manifestations is the anticrossing between the exciton and cavity modes ${ }^{1,3-5}$ resulting in Rabi oscillations between the new eigenstates of the system (termed cavity polaritons). ${ }^{6}$ However, the main difference between strong coupling in atomic and semiconductor physics is the delocalized nature of the eigenstates, which have in-plane dispersion curves due to the twodimensional translational invariance in the layers. ${ }^{7}$ The symmetry of the interacting system leads to a one-to-one correspondence between a polariton wave vector in the cavity and the direction of the emitted photon. This allows one to observe the polariton dispersion curve using angle resolved photoluminescence and reflectivity experiments. ${ }^{4,8}$

In our experiment, we have evidenced a new manifestation of this in-plane dispersion, namely, the propagation of the polaritons in the plane of the microcavity. Since they are created resonantly by a short laser pulse, they are quantum wave packets propagating with a group velocity given by the expression $^{9}$

$$
v_{g}\left(k_{p}\right)=\frac{1}{\hbar} \frac{\partial E\left(k_{p}\right)}{\partial k_{p}},
$$

where $k_{p}$ is the polariton in-plane wave vector and $E\left(k_{p}\right)$ its energy. Due to the strong dispersion of the polariton energy, ${ }^{4,5,7,8}$ this velocity is many orders of magnitude larger than the bare exciton one. Moreover, as a consequence of the mixed state nature of the polariton, the group velocity $v_{g}$ is expected to depend strongly on the detuning $\delta$ between the cavity and exciton energies $\left(\delta=E_{c 0}-E_{x 0}\right)$ and on the incident angle $\theta_{i}$.

Our experiment uses a standard time-resolved luminescence setup (Fig. 1). The excitation source is a Ti-Sa mode locked laser which delivers nearly Fourier transform limited
$1.5 \mathrm{ps}$ long pulses with a repetition rate of $82 \mathrm{MHz}$. The laser beam is focused on a spot of about $50 \mu \mathrm{m}$ waist by a lens of $35 \mathrm{~mm}$ diameter and $80 \mathrm{~mm}$ focal length. The emission from the sample is collimated by the same lens in a parallel beam which is then focused with a times 4 magnification on the vertical entrance slit of a spectrograph. The output slit of the spectrograph is replaced by the $50 \mu \mathrm{m}$ wide horizontal entrance slit of a streak camera that makes a time to vertical displacement conversion so that with a charge-coupled de-

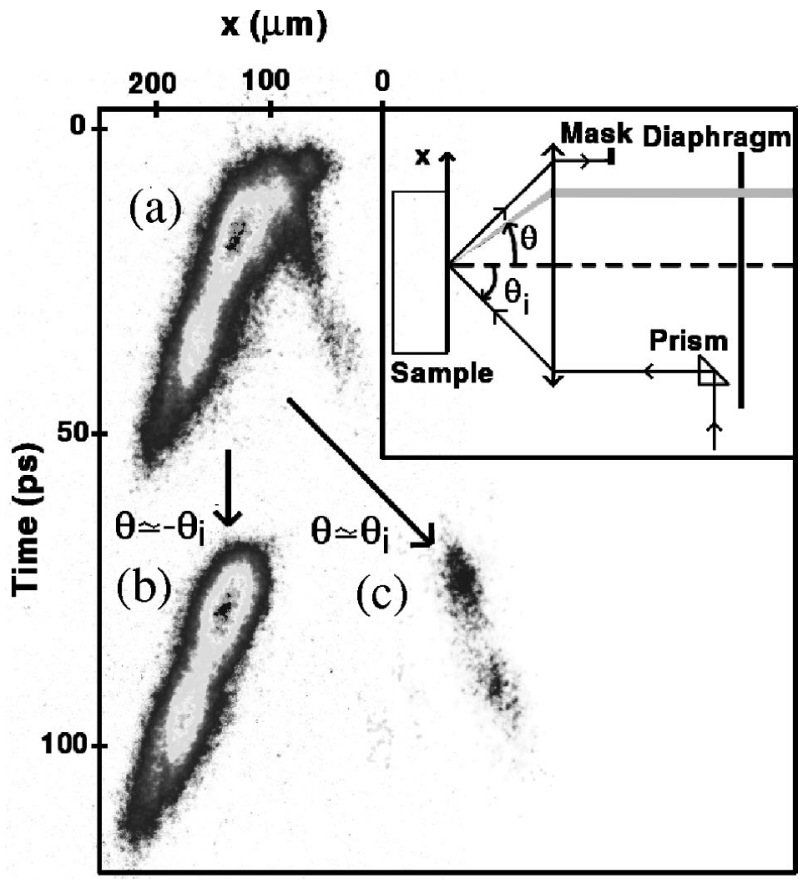

FIG. 1. Propagation in the horizontal direction of the secondary emission source as observed on the streak camera after resonant excitation of the lower branch polariton $\left(\delta=-10 \mathrm{meV}\right.$ and $\theta_{i}$ $=7^{\circ}$ ). (a) corresponds to the global emission without angular selection, (b) to an angular selection close to $-\theta_{i}$ and (c) close to $\theta_{i}$. Inserted is the geometry of the experimental setup. 


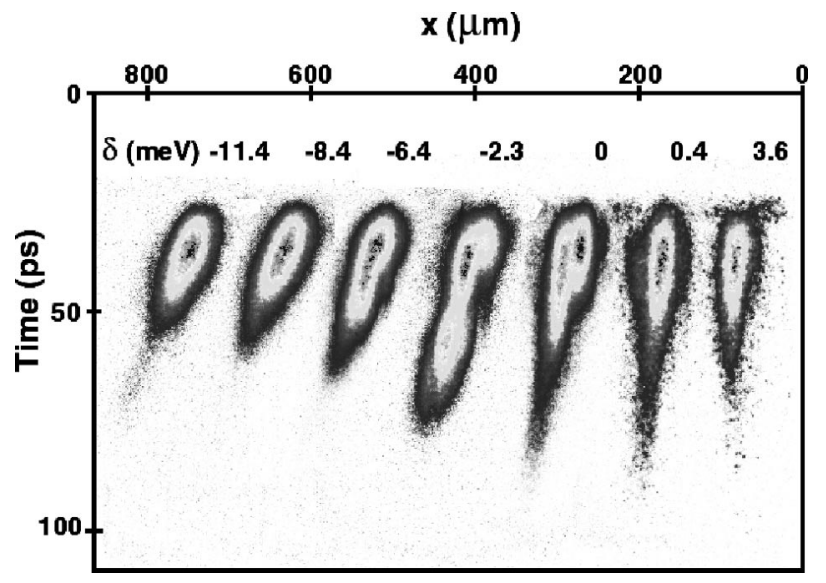

FIG. 2. Propagation in real space of the secondary emission source as observed on the streak camera for several detunings. The diaphragm is close to the reflection and $\theta_{i}=7.8^{\circ}$.

vice (CCD) camera, we get the emission intensity as a function of wavelength and time. The time resolution is about 2.5 ps. When the structure, is resonantly excited, we mask the laser reflection on the sample surface, using a $4 \mathrm{~mm}$ wide mask. If we want to observe the horizontal movement of the excited spot on the sample, we open the entrance slit of the monochromator to the maximum and turn the grating to the zero order so that we get on the monitor the emission intensity as a function of the horizontal position on the sample ( $x$ axis) and time. In fact, we measure the projection of the excited spot movement on the $x$ axis.

The studied sample is a $\lambda$ GaAs microcavity containing an $\mathrm{In}_{0.05} \mathrm{Ga}_{0.95} \mathrm{As} 8 \mathrm{~nm}$ thick quantum well located at the antinode of the electromagnetic field. The Bragg reflectors are made of 24 (substrate side) and 20 (air side) pairs of $\mathrm{AlAs} / \mathrm{Al}_{0.1} \mathrm{Ga}_{0.9} \mathrm{As}$ quarter wavelength layers, giving a cavity photon lifetime of nearly $6 \mathrm{ps}$. The sample is maintained at a temperature of $8 \mathrm{~K}$. All the experiments were done in the low excitation regime, with polariton density between $10^{8} \mathrm{~cm}^{-2}$ and $10^{9} \mathrm{~cm}^{-2}$. When exciting the structure above the cavity stop-band, one can see the luminescence peaks of the lower and upper polariton modes whose energies vary along the sample as in previous papers, ${ }^{5,10}$ showing a Rabi splitting of about $3.45 \mathrm{meV}$. The quality of the structure is evidenced by the spectral width of the lower mode, which is $0.2 \mathrm{meV}$ when the mode is cavitylike $(\delta \sim-10 \mathrm{meV})$ and $0.7 \mathrm{meV}$ when the mode is excitonlike $(\delta \sim+4 \mathrm{meV})$.

The experimental procedure is the following: We resonantly excite the lower polariton mode by looking at the emission spectrum with the monochromator working at first order. Then we turn the grating at zero order to observe the time evolution of the luminescence spot as shown in Fig. 1. We see two symmetric traces. The major one corresponds to the propagation in the same direction as the incident beam. The smaller one goes backward. If we select the polariton in-plane wave vector by inserting an $8 \mathrm{~mm}$ diameter diaphragm in the collimated beam, we see that the trace propagating in the forward direction corresponds to wave vectors close to the reflection of the laser $\left(\theta \sim-\theta_{i}\right)$ and the backward one to wave vectors in the opposite direction $\left(\theta \sim \theta_{i}\right)$. The trace in the forward direction is more intense than all the others. This shows that most of the polaritons keep the same

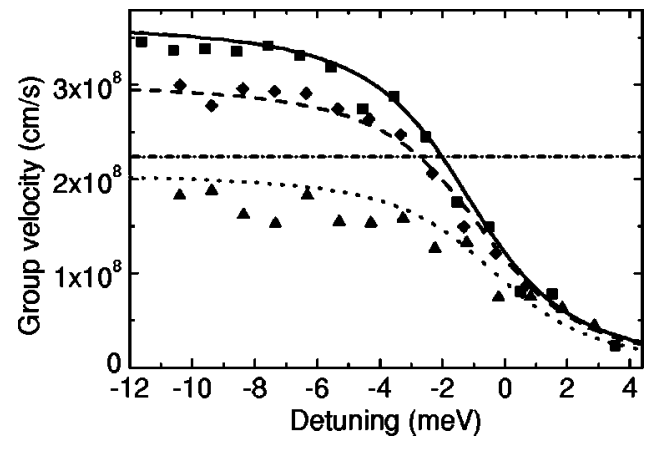

FIG. 3. Group velocity of the resonantly excited lower branch polaritons as a function of the detuning for different incident angles $\theta_{i}$. The points are the measured values for $\theta_{i}=7.8^{\circ}(\boldsymbol{\square}), \theta_{i}$ $=6.2^{\circ}(\diamond)$, and $\theta_{i}=4.3^{\circ}(\boldsymbol{\Delta})$. The solid, dashed, and dotted lines correspond to the group velocities calculated for the previous angles. The dashed-dotted line is the calculated velocity for the transmitted beam through the GaAs substrate and with $\theta_{i}=7.8^{\circ}$.

wave vector as the excitation wave vector. Furthermore, the trace stays nearly on a straight line, meaning that the velocity is constant and that the movement continues in the same direction (if the direction of the movement was changed, the velocity in the $x$ direction would decrease). Since we mask the reflected beam, we can note that most of the measured intensity is due to polaritons that have experienced small momentum scattering.

In Fig. 2, we show the evolution of the propagating trace (for $\theta \sim-\theta_{i}$ ) with the detuning. The slope of the trace recorded by the CCD measures the group velocity of the polaritons. The trace is oblique for large negative detunings (cavitylike polaritons), which is the sign of a large velocity, and becomes progressively vertical when the detuning increases. After calibrating the relation between the displacement on the sample and the number of pixels on the streak camera, we have calculated the measured polariton velocity as a function of the detuning $\delta$ and the incident angle $\theta_{i}$ as shown in Figs. 3 and 4.

The theoretical curves plotted on the same figures are calculated from the polariton dispersion curves. The incident laser beam, making an angle $\theta_{i}$ with the normal to the sample, creates polaritons whose wave vector $\mathbf{k}_{p}$ is equal to the projection on the plane $\mathbf{k}_{i \|}$ of the incident wave vector $\mathbf{k}_{i}$ :

$$
k_{p}=k_{i \|}=k_{i} \sin \theta_{i} .
$$

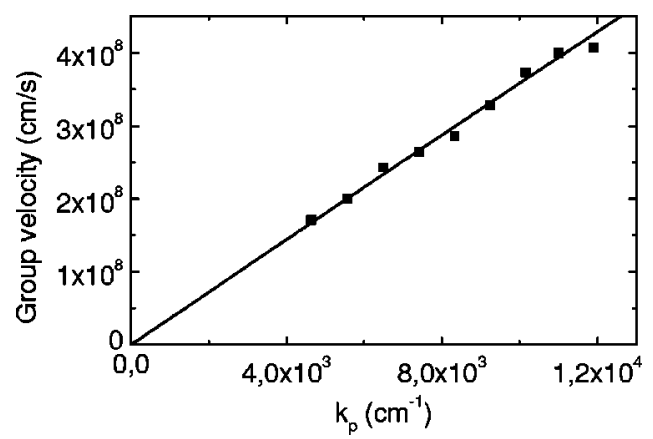

FIG. 4. Variation of the lower branch polariton group velocity as a function of the in-plane wave vector $k_{p}$ for $\delta=-10 \mathrm{meV}$. The points (line) are the measured (calculated) values. 
As long as these polaritons are not scattered, they propagate in a straight line with a velocity given by the expression (1). In a two-oscillator model, ${ }^{5,11}$ the lower polariton energy $E_{l}\left(k_{p}\right)$ is given by

$$
E_{l}\left(k_{p}\right)=\frac{E_{x}\left(k_{p}\right)+E_{c}\left(k_{p}\right)}{2}-\frac{\sqrt{\left[E_{c}\left(k_{p}\right)-E_{x}\left(k_{p}\right)\right]^{2}+\Omega_{R}^{2}}}{2},
$$

where $\Omega_{R}$ is the Rabi splitting and $E_{x}\left(k_{p}\right)$ and $E_{c}\left(k_{p}\right)$ the exciton and cavity energies. The exciton energy dispersion is parabolic: $E_{x}\left(k_{p}\right)=E_{x 0}+\hbar^{2} k_{p}^{2} / 2 M_{x}$ where $M_{x}$ is the exciton effective mass. The cavity mode energy is given by the expression

$$
E_{c}\left(k_{p}\right)=\sqrt{E_{c 0}^{2}+\frac{\hbar^{2} k_{p}^{2} c^{2}}{n_{\mathrm{eff}}^{2}}},
$$

where $n_{\text {eff }}$ is the effective refractive index of the cavity.

The refractive index $n_{\text {eff }}$ is an average, over the spatial extension of the optical cavity mode, of the layer refractive indices at the polariton energy $(\sim 1.472 \mathrm{eV})$. Since, to our knowledge, the refractive indices of GaAs and AlAs have not been measured at $10 \mathrm{~K}$, we consider in first approximation that the low temperature value is equal to the high one at a lower energy corresponding to the band gap variation between these two temperatures. ${ }^{12}$ So we take the following values: $n_{\mathrm{GaAs}}=3.57$ (Ref. 13 at $1.455 \mathrm{eV}$ and $\left.100 \mathrm{~K}\right), n_{\mathrm{AlAs}}$ $=2.965$ (Ref. 14 at $1.38 \mathrm{eV}$ and $300 \mathrm{~K})$, and a linear interpolation for $\mathrm{Al}_{0.1} \mathrm{Ga}_{0.9} \mathrm{As}$. The spatial extension of the optical mode is calculated as in Refs. 15 and 16 by taking into account the effective order $m_{\text {eff }}$ of the cavity mode. Averaging the refractive index $n(z)$ of the structure over the field extension gives $n_{\text {eff }}=3.32$. A more rigorous determination of the cavity mode dispersion using a transfer matrix method for the whole structure ${ }^{17}$ gives approximately the same value. The group velocities calculated with this refractive index are plotted in Figs. 3 and 4. The agreement with the experimental velocities is good (the uncertainty on the experimental values is about $10 \%$ ).

One should also consider the part of the incident beam which is transmitted by the microcavity and goes through the substrate. Since the opposite face of the substrate is not polished, a reflection of the beam by this face is excluded. However, the scattering of the beam by the GaAs substrate would give a trace on the CCD camera with a velocity in the $x$ direction given by $v_{t}=c \sin \theta_{i} / n n_{g}$ where $n$ and $n_{g}$ are the refraction and group optical indices of GaAs. For an incident angle $\theta_{i}=7.8^{\circ}$ and using $n_{g}=5.1,{ }^{13}$ we find $v_{t}=2.24$ $\times 10^{8} \mathrm{~cm} / \mathrm{s}$. This value is different from the experimental ones (Fig. 3) and does not vary with the detuning, excluding this interpretation. Furthermore, we have done the experiment on a microcavity grown on a $2 \times 10^{19} \mathrm{~cm}^{-3} p$-doped substrate. Although the beam transmitted through the substrate is attenuated by at least a factor of 10 on this sample, ${ }^{18}$ we still see the propagation of the emission source, thus confirming our previous analysis.

Let us note that the polariton group velocity (1) can be expressed as a linear combination of the exciton and cavity velocities. Using a simple two-oscillator model, ${ }^{5,11}$ the polar-

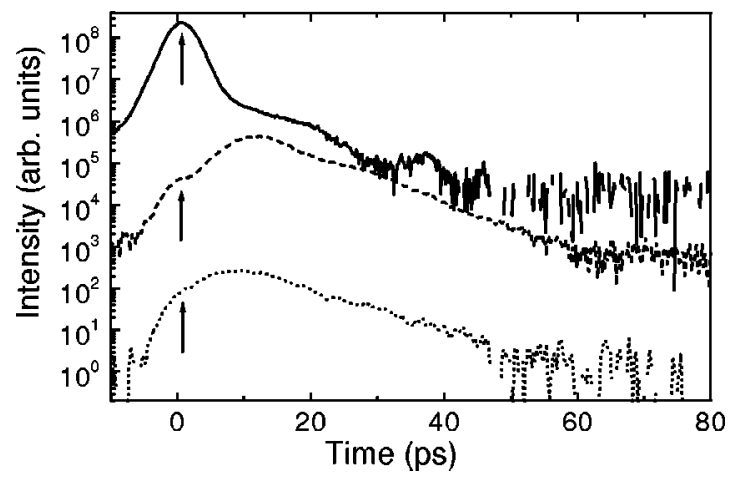

FIG. 5. Temporal evolution of the secondary emission with different angular selection conditions: (a) without diaphragm and laser mask (full lines), (b) with laser mask and without diaphragm (dashed lines), (c) with a diaphragm on the upper part of the elastic ring (dotted lines). The arrows indicate the peak due to the laser reflection and scattering by the sample surface.

iton wave function $\left|L_{k_{p}}\right\rangle$ can be expressed as a linear combination of the exciton and photon wave functions,

$$
\left|L_{k_{p}}\right\rangle=c_{k_{p}}\left|C_{k_{p}}\right\rangle+x_{k_{p}}\left|X_{k_{p}}\right\rangle,
$$

where $c_{k_{p}}\left(x_{k_{p}}\right)$ is the cavity weight (exciton weight) of the polariton state. Using the polariton group velocity definition (1) together with the energy dispersion (3) we find

$$
v_{g}\left(k_{p}\right)=x_{k_{p}}^{2} v_{x}\left(k_{p}\right)+c_{k_{p}}^{2} v_{c}\left(k_{p}\right),
$$

where $v_{x}\left(k_{p}\right)$ and $v_{c}\left(k_{p}\right)$ are the group velocities of the uncoupled cavity and exciton modes. Though photons are not massive particles, the cavity energy dispersion (4) can be approximated, for $k_{p} \ll 2 \pi / L_{c}$, by

$$
E_{c}\left(k_{p}\right) \sim E_{c 0}+\frac{\hbar^{2} k_{p}^{2}}{2 M_{c}}
$$

where $M_{c}=n_{\mathrm{eff}}^{2} E_{c 0} / c^{2}$ is a kind of effective mass of the cavity photon $\left(M_{c}=3 \times 10^{-5} m_{0}\right.$ in our case $) .{ }^{19}$ The polariton mode still has a parabolic energy dispersion at small $k_{p}$ with an effective mass $M_{l}$ varying between $M_{c}$ and $M_{x}\left(M_{x} \sim 0.3 m_{0}\right)$. For example, in Fig. 4, corresponding to the case of a cavitylike polariton $\left(\delta \ll-\Omega_{R}\right)$, the linear variation of the group velocity with the wave vector leads to a parabolic dispersion curve with an effective mass $M_{p}$ $=3.2 \times 10^{-5} m_{0}$ very close to that of the cavity photon. We are then faced with quasiparticles whose mass can be varied by four orders of magnitude just by varying the detuning. ${ }^{19}$

Finally, we would like to discuss an important aspect of our observations, namely, the conservation of the polariton in-plane wave vector during all its propagation. In Fig. 5 we show the temporal evolution of the secondary emission at negative detuning $(\delta=-5 \mathrm{meV})$ with different angular selections on another sample. The decay time of the secondary emission is $7.5 \mathrm{ps}$, which is close to the calculated photon lifetime in this structure. Also reported is the temporal evolution of the emission from the upper part of the elastic ring (polaritons with $\left.k_{p}=k_{i \|}\right) .{ }^{20}$ The comparison between these spectra indicates that most of the emission arises from polaritons that have not been scattered: the intensity on the 
elastic ring is indeed four orders of magnitude smaller than that recorded at the reflection. This is not surprising at negative detuning (photonlike polariton) where scattering is strongly reduced due to the weak exciton part in the polariton wave function. When the detuning is increased, both elastic and inelastic scattering should become more efficient since the exciton weight increases. But, as seen in Fig. 2, we do not observe a break in the ballistic propagation of the polaritons when the detuning is increased: the excited polaritons propagate ballistically during their lifetime, thus conserving their in-plane wave vector. This is an important result of this paper. As previously proposed by Whittaker et al., ${ }^{19}$ Savona et al., ${ }^{21}$ and Baumberg et al., ${ }^{22}$ it is due to the fact that the resonantly excited polaritons have a small wave vector $\left(\leqslant 10^{4} \mathrm{~cm}^{-2}\right)$ and a large wavelength $(\geqslant 8 \mu \mathrm{m})$. Thus, they are little scattered by the quantum well interface rugosity whose typical lengths are close to $0.1 \mu \mathrm{m}$. Furthermore, due to the cavity coupling, the density of states is small, which makes the scattering to other states at the same energy less efficient. ${ }^{23}$ Let us note that similar long dephasing times have been observed by Cassabois et al. ${ }^{24}$ close to the anticrossing.

We would like to thank V. Thierry-Mieg for the growth of the sample and J. M. Gerard, C. Tanguy, I. Sagnes, AllokRudra, J. L. Oudar, L. C. Andreani, and G. R. Hayes for fruitful discussions.
${ }^{1}$ C. Weisbuch, M. Nishioka, A. Ishikawa, and Y. Arakawa, Phys. Rev. Lett. 69, 3314 (1992).

${ }^{2} \mathrm{~S}$. Haroche, Fundamental Systems in Quantum Optics (Elsevier, Amsterdam, 1992).

${ }^{3}$ R. Houdré, R. P. Stanley, U. Oesterle, and M. Ilegems, Phys. Rev. B 49, 16761 (1994).

${ }^{4}$ R. Houdré et al., Phys. Rev. Lett. 73, 2043 (1994).

${ }^{5}$ B. Sermage, Ann. Phys. C2 Suppl. 3 20, 297 (1995); B. Sermage et al., Phys. Rev. B 53, 16516 (1996).

${ }^{6}$ T. B. Norris et al., Phys. Rev. B 50, 14663 (1994).

${ }^{7}$ S. Jorda, Phys. Rev. B 51, 10185 (1995).

${ }^{8}$ D. Baxter et al., Phys. Rev. B 56, 10032 (1997).

${ }^{9}$ O. Madelung, Introduction to Solid-State Theory (Springer, Berlin, 1996).

${ }^{10}$ R. P. Stanley et al., Phys. Rev. B 53, 10995 (1996).

${ }^{11} \mathrm{~V}$. Savona et al., New Aspects in Optical Properties of Nanostructures, Phase Transitions (Gordon and Breach, New York, 1998).
${ }^{12}$ Numerical Data and Functional Relationships in Science and Technology, edited by O. Madelung, Landolt-Börnstein, Group III, Vol. 17, pt. a. (Springer, Berlin, 1982).

${ }^{13}$ D. T. F. Marple, J. Appl. Phys. 35, 1241 (1964).

${ }^{14}$ B. Monemar, Solid State Commun. 8, 2121 (1970).

${ }^{15}$ R. P. Stanley et al., Appl. Phys. Lett. 65, 1883 (1994).

${ }^{16}$ G. Panzarini et al., Phys. Rev. B 59, 5082 (1999).

${ }^{17}$ M. R. Vladimirova, A. V. Kavokin, and M. A. Kaliteevski, Phys. Rev. B 54, 14566 (1996).

${ }^{18}$ D. I. Babic, Ph.D. thesis, University of California at Santa Barbara, 1995.

${ }^{19}$ D. M. Whittaker et al., Phys. Rev. Lett. 77, 4792 (1996).

${ }^{20}$ T. Freixanet et al., Phys. Rev. B 60, 8509 (1999).

${ }^{21}$ V. Savona et al., Phys. Rev. Lett. 78, 4470 (1997).

${ }^{22}$ J. J. Baumberg, A. Armitage, M. S. Skolnick, and J. S. Roberts, Phys. Rev. Lett. 81, 661 (1998).

${ }^{23}$ C. Ciuti et al., Phys. Rev. B 58, 10123 (1998).

${ }^{24}$ G. Cassabois et al., Phys. Rev. B 59, 10429 (1999). 